\title{
Epithelioid hemangioendotheliomas with TFE3 gene translocations are compossible with CAMTA1 gene rearrangements
}

\author{
Seok Joo Lee ${ }^{1}$, Woo Ick Yang ${ }^{1}$, Woo-Suk Chung ${ }^{2}$ and Sang Kyum Kim ${ }^{1}$ \\ ${ }^{1}$ Department of Pathology, Yonsei University Medical Center, Seoul, South Korea \\ 2 Department of Diagnostic Radiology, Konyang University Hospital, Daejeon, South Korea \\ Correspondence to: Sang Kyum Kim, email: nicekyumi@yuhs.ac \\ Keywords: epithelioid hemangioendothelioma, TFE3, YAP1, CAMTA 1, WWTR 1, Pathology Section \\ Received: September 02,2015 Accepted: January 21,2016 Published: January 28, 2016
}

\section{ABSTRACT}

Epithelioid hemangioendotheliomas (EHEs) are vascular tumors of intermediate malignancy that can undergo high-grade malignant transformations. EHEs have been characterized by tumor-specific WW domain-containing transcription regulator 1(WWTR1)-calmodulin-binding transcription activator 1 (CAMTA1) translocations, and recently, a novel Yes-associated protein 1 (YAP1)-transcription factor E3 (TFE3) gene fusion was identified in EHEs. In this study, we examined the expression levels of TFE 3 and CAMTA1 via immunohistochemical staining and identified chromosomal alterations using fluorescence in situ hybridization (FISH) assays and RT-PCR tests. Although all of the EHEs were CAMTA1-positive in immunohistochemical staining, only five out of 18 EHEs ( $27.78 \%$ ) positively expressed nuclear TFE3. The five TFE3-positive EHEs exhibited TFE3 gene break-apart in FISH assays. YAP1-TFE3 gene fusions were confirmed by RT-PCR. Interestingly, we observed CAMTA1 gene break-apart in all of the five TFE3-positive EHEs via FISH assays, and four out of the five TFE3-positive EHEs exhibited WWTR1-CAMTA1 gene fusions via RT-PCR. These results indicate that these two chromosomal alterations are not mutually exclusive but compossible in EHEs. Finally, primary tumor sites in TFE3-positive EHEs consistently contained single masses $(P=0.0359)$ with larger sizes $(P=0.0550)$ compared to TFE3-negative EHEs. Similar to previous reports, we observed well-formed vessels more frequently in TFE3-positive EHEs than in TFE3-negative EHEs $(P=\mathbf{0 . 0 4 4 1})$. In addition, TFE3positive EHEs tended to more frequently demonstrate high-grade nuclear atypia ( $P$ $=0.0654)$ and hypercellularity $(P=0.0987)$ than TFE3-negative EHEs. Thus, we have now established two clinically distinct subgroups of EHEs: TFE3-positive and TFE3negative EHEs.

\section{INTRODUCTION}

Epithelioid hemangioendotheliomas (EHEs) are malignant angiocentric vascular neoplasms composed of chains and cords of epithelioid endothelial cells distributed in a myxohyaline stroma [1]. EHEs affect patients of all ages and arise in virtually any body site. High-risk patients with large tumor sizes and frequent mitosis have a poorer disease-specific survival of $59 \%$ compared to $100 \%$ survival of patients whose tumors lacked these features [2].

Several studies have reported an EHE-specific translocation resulting in the fusion of the WW domain- containing transcription regulator 1 (WWTR1) gene on 3q23-24 with the calmodulin-binding transcription activator 1 (CAMTA1) gene on 1p36 [3-5]. Recently, researchers identified a novel fusion between the Yesassociated protein $1(Y A P 1)$ gene and the transcription factor E3 (TFE3) gene, which defined a distinct subset of EHEs characterized by somewhat different histological morphologies, including focally well-formed vascular channels and variably solid architectures, clinically occurred in young adults $[6,7]$.

However, it remains unclear if these two distinct chromosomal alterations, the WWTR1-CAMTA1 fusion and the YAP1-TFE3 fusion specifically, are mutually 
Table 1: Clinical presentation of EHEs

\begin{tabular}{|c|c|c|c|c|c|c|c|c|}
\hline Case & Sex/Age & Primary site & \begin{tabular}{|l|} 
Tumor \\
size $^{\mathrm{a}}$ \\
$(\mathrm{cm})$ \\
\end{tabular} & Multiplicity & $\begin{array}{l}\text { Metastatic } \\
\text { site }\end{array}$ & Treatment & Recurrence $^{c}$ & \begin{tabular}{|l|}
$\begin{array}{l}\text { Survival } \\
\text { (follow-up } \\
\text { month) }\end{array}$ \\
\end{tabular} \\
\hline 1 & $\mathrm{M} / 59$ & Tongue & $-{ }^{d}$ & Single & - & STx & No & Alive (241) \\
\hline 2 & $\mathrm{M} / 26$ & Liver & 1.0 & Multiple & Lung & STx, CTx & No & Alive (240) \\
\hline 3 & $\mathrm{M} / 46$ & Inguinal area & 2.0 & Multiple & \begin{tabular}{|l|} 
Lung, lymph \\
node, brain, \\
breast
\end{tabular} & STx & No & Dead (7) \\
\hline 4 & $\mathrm{M} / 42$ & Liver & 10.7 & Single & Lung & STx, TACE & No & Alive (145) \\
\hline 5 & $\mathrm{M} / 46$ & Liver & 1.6 & Multiple & - & STx & No & Alive (105) \\
\hline 6 & $F / 39$ & Lung & 2.8 & Single & - & STx & No & Alive (95) \\
\hline 7 & $\mathrm{~F} / 54$ & Thigh & 19.0 & Single & - & STx, RTx & No & Alive (83) \\
\hline 8 & $\mathrm{~F} / 57$ & Axilla & 1.5 & Single & - & STx & No & Alive (75) \\
\hline 9 & $\mathrm{~F} / 82$ & Liver & 10.8 & Multiple & - & STx, TACE & No & Alive (73) \\
\hline 10 & $\mathrm{~F} / 37$ & Liver & 4.2 & Multiple & Lung & STx, CTx & No & Alive (67) \\
\hline 11 & $F / 35$ & Liver & 3.3 & Multiple & - & STx & No & Alive (59) \\
\hline 12 & $\mathrm{~F} / 55$ & Upper arm & 1.9 & Single & Lung & STx, CTx & Yes & Alive (58) \\
\hline 13 & $\mathrm{M} / 51$ & Liver & 4.0 & Multiple & - & STx & No & Alive (42) \\
\hline 14 & $\mathrm{~F} / 23$ & Submandible & 1.5 & Single & - & STx & No & Alive (40) \\
\hline 15 & $\mathrm{M} / 70$ & Lung & 6.0 & Single & $\begin{array}{l}\text { Lymph node, } \\
\text { liver, adrenal } \\
\text { gland }\end{array}$ & STx, RTx & No & Dead (2) \\
\hline 16 & $\mathrm{~F} / 69$ & Parapharynx & 3.8 & Single & - & STx & Yes & Alive (33) \\
\hline 17 & $\mathrm{M} / 48$ & Femur & 7.2 & Single & \begin{tabular}{|l} 
Stomach, \\
lung \\
\end{tabular} & $\begin{array}{ll}\text { STx, } & \text { CTx, } \\
\text { RTx } & \\
\end{array}$ & No & Alive (32) \\
\hline 18 & $\mathrm{~F} / 59$ & Liver & 1.2 & Multiple & Lung & STx & No & Alive (6) \\
\hline
\end{tabular}

exclusive in EHEs. In this study, we assess TFE3 rearrangements in EHEs to verify the newly proposed classification system for EHEs and examine the details of the clinicopathological characteristics of TFE3 rearranged EHEs.

\section{RESULTS}

\section{Clinicopathologic data of EHEs}

A total of 18 patients with EHEs (eight men and ten women) were included in the study, and the age at diagnosis ranged between 23-82 years (mean \pm standard deviation: $49.89 \pm 15.26$ ) (Table 1). Tumors of various sizes $(4.85 \pm 4.77 \mathrm{~cm})$ arose from a variety of organs. Eight out of 18 EHEs (44.4\%) occurred in the liver, and tumors most frequently spread to the lungs $(7 / 8,87.5 \%)$. 
Table 2: Histologic findings and immunohistochemical staining results of EHEs

\begin{tabular}{|c|c|c|c|}
\hline Case & Histologic feature & $\begin{array}{l}\text { CAMTA1 } \\
\text { expression }\end{array}$ & TFE3 expression \\
\hline 1 & Spindle cell feature, foamy cytoplasm & + & - \\
\hline 2 & Abundant stroma & + & - \\
\hline 3 & Abundant stroma & + & - \\
\hline 4 & Abundant stroma & + & + \\
\hline 5 & Abundant stroma & + & - \\
\hline 6 & $\begin{array}{l}\text { Well-formed blood vessels, spindle cell feature, foamy } \\
\text { cytoplasm, mitotic activity (2/10 HPFs), hypercellularity }\end{array}$ & + & + \\
\hline 7 & $\begin{array}{l}\text { Well-formed blood vessels, spindle cell feature, abundant } \\
\text { stroma, focal high-grade nuclear atypia, hypercellularity }\end{array}$ & + & + \\
\hline 8 & $\begin{array}{l}\text { Well-formed blood vessels, mitotic activity (1/10 HPFs), } \\
\text { hypercellularity }\end{array}$ & + & - \\
\hline 9 & $\begin{array}{l}\text { Abundant stroma, mitotic activity (1/10 HPFs), tumor } \\
\text { necrosis }\end{array}$ & + & - \\
\hline 10 & Abundant stroma, tumor necrosis & + & - \\
\hline 11 & Abundant stroma & + & - \\
\hline 12 & $\begin{array}{l}\text { Spindle cell feature, abundant stroma, mitotic activity } \\
(1 / 10 \text { HPFs })\end{array}$ & + & - \\
\hline 13 & $\begin{array}{l}\text { Abundant stroma, mitotic activity (1/10 HPFs), tumor } \\
\text { necrosis }\end{array}$ & + & - \\
\hline 14 & $\begin{array}{l}\text { Abundant stroma, mitotic activity (1/10 HPFs), tumor } \\
\text { necrosis }\end{array}$ & + & + \\
\hline 15 & Spindle cell feature, abundant stroma, foamy cytoplasm & + & - \\
\hline 16 & $\begin{array}{l}\text { Spindle cell feature, foamy cytoplasm, mitotic activity } \\
(1 / 10 \text { HPFs })\end{array}$ & + & - \\
\hline 17 & $\begin{array}{l}\text { Abundant stroma, moderate to focal high-grade nuclear } \\
\text { atypia, foamy cytoplasm, mitotic activity (1/10 HPFs), } \\
\text { tumor necrosis }\end{array}$ & + & + \\
\hline 18 & Abundant stroma & + & - \\
\hline Total & & $18 / 18(100.0 \%)$ & $5 / 18(27.8 \%)$ \\
\hline
\end{tabular}

At diagnosis, eight out of 18 EHEs (44.4\%) presented as multiple nodules in the primary lesion. Two out of 18 patients of EHE had tumor recurrence (11.1\%) during follow-up periods $(78.11 \pm 16.36$ months $)$.

Upon microscopic examination, many cases were composed of chains and cords of epithelioid tumor cells distributed in a myxohyaline stroma (Figure 1, Supplementary Data 1). All of the tumors showed strong expression of CD31 or CD34 via immunohistochemistry, indicating that they were vascular tumors. Taken together, the diagnoses of these cases were consistent with EHE.

Next, we performed immunohistochemical staining of CAMTA1 and TFE3 (Figure 1 and Table 2). As a result, all of the EHEs demonstrated strong nuclear staining of CAMTA1. Additionally, TFE3 nuclear expression in tumor cells was identified in five out of 18 cases $(27.8 \%)$.
As previously described $[6,7,11]$, we also found wellformed vascular channels in the five TFE3-positive EHEs on a microscopic examination. Because CAMTA1 expressions were also observed in the five TFE3-positive EHEs at the same time, we decided that other tests were necessary to determine expression status of CAMTA1 and TFE3 in EHEs.

\section{YAP1-TFE3 gene fusions are compatible with WWTR1-CAMTA1 translocations in EHEs}

To verify TFE3 gene status, we performed FISH assays using TFE3 break-apart probes. We confirmed the presence of TFE3 gene translocations in the five EHEs that were immunohistochemically TFE3-positive (Figure 
Table 3: Chromosomal alteration of TFE3 and CAMTA1

\begin{tabular}{|c|c|c|c|c|}
\hline \multirow{2}{*}{ Case } & \multicolumn{2}{|c|}{ TFE3 status } & \multicolumn{2}{|c|}{$C A M T A 1$ status } \\
\hline & FISHa $^{a}$ & RT-PCR $^{\text {b }}$ & FISH $^{\mathrm{c}}$ & RT-PCR ${ }^{d}$ \\
\hline 4 & + & Unknown $^{\mathrm{e}}$ & + & Unknown ${ }^{\mathrm{e}}$ \\
\hline 6 & + & + & + & + \\
\hline 7 & + & + & + & + \\
\hline 14 & + & + & + & + \\
\hline 17 & + & + & + & + \\
\hline \multicolumn{5}{|c|}{$\begin{array}{l}\text { a: Interpreted as positive }(+) \text { when at least } 10 \text { out of } 50 \text { tumor cells }(20 \%) \\
\text { showed separate green and orange signals using TFE3 break-apart probes. } \\
\text { b: Interpreted as positive }(+) \text { when the YAPI-TFE3 fusion transcript was } \\
\text { observed via RT-PCR. } \\
\text { c: Interpreted as positive }(+) \text { when at least } 10 \text { out of } 50 \text { tumor cells }(20 \%) \\
\text { showed separate green and orange signals using CAMTA1 break-apart probes. } \\
\text { d: Interpreted as positive }(+) \text { when the WWTR1-CAMTA1 fusion transcript was } \\
\text { observed via RT-PCR. } \\
\text { e: Value could not be determined because RNA extraction was failed. }\end{array}$} \\
\hline
\end{tabular}

Table 4: Comparison of the clinical presentations of TFE3-positive and TFE3-negative EHEs

\begin{tabular}{|l|l|l|l|}
\hline$(\%)$ & TFE3 $(+)$ EHE & TFE3 (-) EHE & $P$-value \\
\hline Case number & 5 & 13 & \\
\hline Age at diagnosis (year) & $41.20 \pm 5.23$ & $53.23 \pm 4.30$ & 0.1383 \\
\hline Sex (Male:Female) & $2: 3$ & $6: 7$ & 1.0000 \\
\hline Follow-up period (month) & $79.00 \pm 20.44$ & $77.77 \pm 37.66$ & 0.9743 \\
\hline Overall survival & $5 / 5(100.0)$ & $11 / 13(84.6)$ & $0.3598^{\text {a }}$ \\
\hline Tumor size (cm) & $8.24 \pm 3.14$ & $3.44 \pm 0.80$ & 0.0550 \\
\hline Multiplicity & $0 / 5(0.0)$ & $8 / 13(61.5)$ & 0.0359 \\
\hline Local Recurrence ${ }^{\text {b }}$ & $0 / 5(0.0)$ & $2 / 13(15.4)$ & 1.0000 \\
\hline \begin{tabular}{l} 
Distant Metastasis \\
\hline $\begin{array}{l}\text { a: } \text { A statistical difference of overall survival between patients with TFE3-positive and TFE3-negative EHEs } \\
\text { by the Kaplan-Meier analysis } \\
\text { b: Tumor recurrence in EHE patients was observed over a follow-up period of two years. }\end{array}$
\end{tabular} \\
\hline
\end{tabular}

2A, upper panels). To ensure the presence of YAP1-TFE3 gene fusions, we performed RT-PCR using a $Y A P 1$ exon 1 forward primer and a TFE3 exon 6 reverse primer. YAP1TFE3 fusion transcripts were identified in four out of five TFE3-positive cases (Figure 2B and Table 3).

In parallel with the YAP1-TFE3 gene fusion assessments, we examined CAMTA1 gene status. As before, we performed FISH assays using CAMTA1 break-apart probes in five EHEs that were immunohistochemically TFE3-positive (Figure 2A, lower panels). Unlike previous reports which claimed mutually exclusive occurrence of WWTR1-CAMTA1 translocation and YAP1-TFE3 gene fusion [6, 7, 11], tumor cells from the TFE3-positive EHEs showed separate signals, indicating the presence of CAMTA1 translocations. We performed RT-PCR using a WWTR1 exon 4 forward primer and a CAMTA1 exon 9 reverse primer, and, interestingly, four TFE3-positive EHEs produced WWTR1-CAMTA1 fusion transcripts (Figure 2B and Table 3). Unfortunately, we were unable to extract RNA in one last case; thus, we could not evaluate the presence of YAP1-TFE3 or WWTR1-CAMTA1 fusions via RT-PCR tests in this case.

Next, we examined the 13 EHEs that were immunohistochemically TFE3 negative. FISH revealed 
that break apart occurred only within the CAMTA1 gene but not the TFE3 gene in these patients (Supplementary Data 2A). We repeatedly attempted to extract mRNA from these patients to perform RT-PCR but failed in 7 of 13 TFE3-negative EHE cases presumably due to mRNA degradation of the formalin-fixed, paraffin-embedded samples. RT-PCR of the other six cases from which we successfully extracted mRNA supported the presence of fusion transcripts of WWTR1-CAMTA1 genes but not YAP1-TFE3 genes (Supplementary Data 2B).

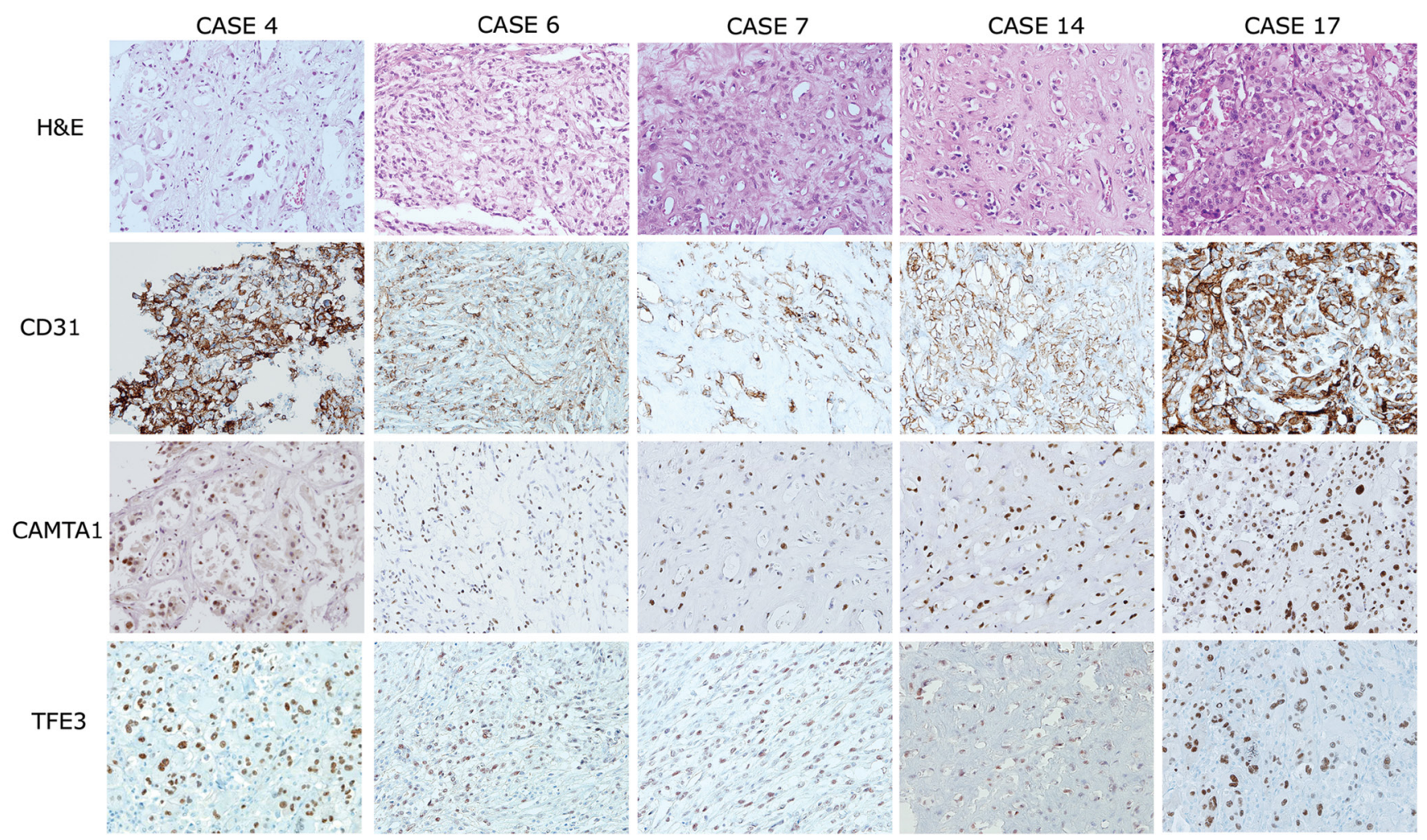

Figure 1: Histological features and immunohistochemical expression profiles of TFE3-positive EHEs. EHEs composed of vasoformative CD31-positive epithelioid tumor cells in a myxohyalinestroma (upper two lanes). Five out of 18 EHEs demonstrated strong simultaneous nuclear expression of CAMTA1 and TFE3 (lower two lanes, respectively). 200× magnification.

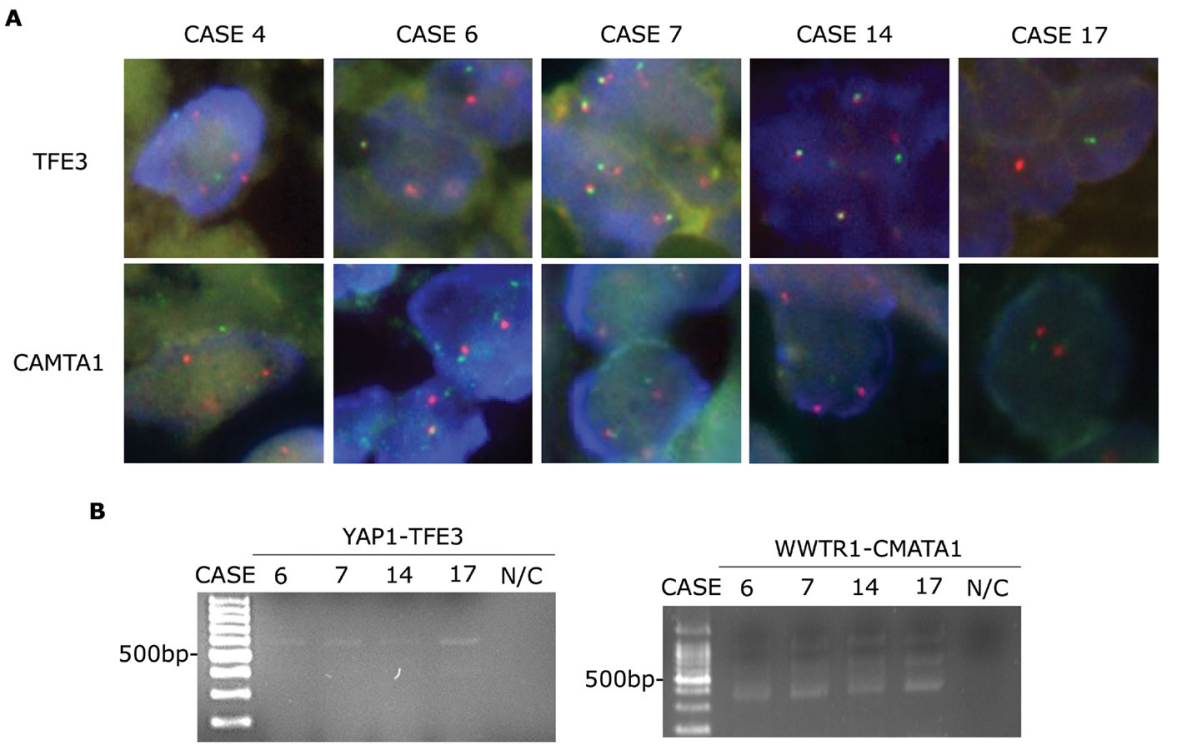

Figure 2: CAMTA1 translocation in EHEs with TFE3 rearrangements. A. FISH assay results for $T F E 3$ and $C A M T A 1$ breakapart showing separated green and orange signals (1,000× magnification). B. RT-PCR assay results identifying YAP1-TFE3 (556 bp, left panel) and WWTR1-CAMTA1 fusion transcripts (400 bp, right panel). N/C, negative control. 
Table 5: Comparison of the histologic features of TFE3-positive and TFE3-negative EHEs

\begin{tabular}{|l|l|l|l|}
\hline$(\%)$ & TFE3 $(+)$ EHE & TFE3 (-) EHE & $P$-value \\
\hline Case number & 5 & 13 & \\
\hline Presence of spindle cytologic feature & $2 / 5(40.0)$ & $4 / 13(30.8)$ & 1.0000 \\
\hline Presence of foamy/feathery cytoplasm & $2 / 5(40.0)$ & $4 / 13(30.8)$ & 1.0000 \\
\hline Presence of high-grade nuclear atypia & $2 / 5(40.0)$ & $0 / 13(0.0)$ & 0.0654 \\
\hline Presence of mitosis & $3 / 5(60.0)$ & $5 / 13(38.5)$ & 0.6078 \\
\hline Presence of hypercellularity & $3 / 5(60.0)$ & $2 / 13(15.4)$ & 0.0987 \\
\hline Presence of well-formed blood vessels & $3 / 5(60.0)$ & $1 / 13(7.7)$ & 0.0441 \\
\hline Abundant stroma & $3 / 5(60.0)$ & $9 / 13(69.2)$ & 1.0000 \\
\hline Tumor necrosis & $2 / 5(40.0)$ & $3 / 13(23.1)$ & 0.5827 \\
\hline
\end{tabular}

\section{Comparison of the clinical and histologic presentation of TFE3-positive and TFE3-negative EHEs}

Using immunohistochemistry, the clinical features of five TFE3-positive EHEs were compared to those of 13 TFE3-negative EHEs (Table 4). At diagnosis, patients with TFE3-positive EHEs tended to be younger (41.20 \pm 5.30 years) than patients with TFE3-negative EHEs (53.23 \pm 4.30 years, $P=0.1383$ ). Additionally, the longest diameter of single tumor size was much larger in TFE3positive EHEs $(8.24 \pm 3.14 \mathrm{~cm})$ compared with TFE3negative EHEs $(3.44 \pm 0.80 \mathrm{~cm}, P=0.0550)$. Although TFE3-negative EHEs demonstrated small tumor sizes, they were more likely to present multiple lesions in the primary tumor site whereas TFE3-positive EHEs exhibited a single lesion $(P=0.0359)$.

In the TFE3-positive group, no local recurrence $(0.0 \%, n=0 / 5)$ occurred, but two distant metastases $(40.0 \%, n=2 / 5)$ developed. Meanwhile, in the TFE3negative group, two local recurrences $(15.4 \%, n=2 / 13)$, and six distant metastases $(53.9 \%, n=6 / 13)$ developed. No statistically significant difference was observed in the rate of local recurrence $(P=1.0)$ and distant metastatic $(P=1.0)$ between TFE3-positive and TFE3-negative EHEs (Table 4). When we compared the overall survival between patients with TFE3-positive and TFE3-negative EHEs by Kaplan-Meier analysis, there was no statistically significant difference (Supplementary Data 3; $P=0.3598$ ).

We compared the histologic features of EHE cases according to their TFE3 expression state but observed varying degrees of myxohyalinized stroma, tumor necrosis, mitotic activity, spindle and epithelioid cytologic features, and foamy and eosinophilic cytoplasm that seemed irrelevant to their TFE3 expression $(P>0.05$, Table 5 and Supplementary Data 1$)$. Similar to previous reports [6,7], we observed well-formed vessels more frequently in TFE3-positive EHEs than in TFE3-negative
EHEs $(P=0.0441)$. In addition, TFE3-positive EHEs tended to more frequently demonstrate high-grade nuclear atypia $(P=0.0654)$ and hypercellularity $(P=0.0987)$ than TFE3-negative EHEs, although the difference did not reach statistical significance.

\section{DISCUSSION}

In this study, we examined the TFE 3 and CAMTA1 protein expression profiles via immunohistochemistry and assessed the chromosomal alterations of TFE3 and CAMTA1 via FISH assays in EHEs. We also verified the presence of YAP1-TFE3 and WWTR1-CAMTA1 fusion transcripts by RT-PCR. These results indicate that these two chromosomal alterations were not mutually exclusive but compossible in EHEs. Therefore, we classified EHEs into two subgroups: TFE3-positive and TFE3-negative EHEs, and compared the clinical and histologic features of each. Notably, TFE3-positive EHEs seemed to present as a single larger mass compared with TFE3-negative EHEs. Similar to a previous report [6], we also observed an earlier onset of disease in patients with TFE3-positive EHEs than in patients with TFE3-negative EHEs and well-formed vascular channels in TFE3-positive EHEs on histological examination. In addition, we observed a tendency for TFE3-positive EHEs to more frequently demonstrate high-grade nuclear atypia and hypercellularity than TFE3-negative EHEs.

A previous study examined CAMTA1 expression profiles in EHEs and identified strong nuclear expression in most cases [8]. Consistent with these findings, we also demonstrated strong nuclear expression of CAMTA1 in $100.0 \%$ of EHEs. In the current study, we were able to identify TFE3 nuclear expression in five out of 18 cases (27. 8\%). This ratio is much lower compared with that of a previous article which claimed to have observed TFE3 nuclear reaction in as much as $87.5 \%(n=21 / 24)$ in their EHEs study population [7]. Our data also differs in that they reported TFE3 rearrangement to occur in merely two 
cases $(5.7 \%, n=2 / 35)$ [7], whereas we detected YAP1TFE3 gene fusion in $22.2 \%(n=4 / 18)$ of EHEs and $80.0 \%$ $(n=4 / 5)$ of TFE3-positive EHEs based on FISH and RTPCR results. EHE remains an extremely rare disease entity of which TFE3 expression has been scarcely examined. Although we add our current observations to the limited literature covering this subject, we acknowledge that subsequent studies which cover more cases are still required to further clarify such discrepancies, especially on TFE3 expression profile and YAP1-TFE3 gene fusion status that exist between our data and the previous report [7].

WWTR1 and YAP1 share conserved amino acid sequences for the WW domain that can interact with PDZ domains. They are named for the shared homology observed in postsynaptic density protein 95 (PSD95), Drosophila discs large tumor suppressor (Dlg1), and zonula occludens-1 protein (zo-1) [9]. Recently, a study described the mechanism of action of a WWTR1(TAZ)CAMTA1 fusion oncoprotein, and the results suggested that the WWTR1-CAMTA1 fusion might cause resistance to anoikis and the oncogenic transformation of tumor cells [10]. This study implies that the WWTR1(TAZ)-CAMTA1 fusion oncoprotein might have a profound role in the molecular pathogenesis of EHEs. Despite the careful analyses reported in previous studies $[6,7,11]$, questions still remain regarding how two very similar molecules could have different translocation partners in one specific tumor type. This concept will be examined in more detail in future studies.

In this study, we observed that TFE3-positive EHEs had a tendency to present as a single larger mass in younger patients compared with TFE3-negative EHEs and demonstrated histologic findings of the presence of high-grade nuclear atypia and hypercellular areas. We believe that these clinicopathologic findings in EHEs with both WWTR1-CAMTA1 and YAP1-TFE3 gene translocations, which were not identified in EHEs having only the WWTR1-CAMTA1 translocation, might reflect a higher degree of genetic heterogeneity in these tumors. Traditionally, sarcomas have been classified into two broad categories: tumors with simple genetic alterations and tumors with complex and unbalanced karyotypes [12]. Tumors with complex genetic alterations are typified by genome instability, resulting in multiple genomic aberrations in the genome of a single tumor and the heterogeneity of aberrations across tumors of a given type $[13,14]$. Although two distinct chromosomal alterations specific to EHEs have been identified, we presume that these two genetic alterations can occur simultaneously in EHEs, possibly reflecting an increased degree of genetic instability.

According to a previous study, tumor size was associated with decreased survival of EHE patients and tumors with $>3$ mitotic figures $/ 50$ high power fields and size $>3.0 \mathrm{~cm}$ had the worst prognosis [2]. Interestingly, we observed that TFE3-positive EHEs presented as single masses with significantly larger sizes compared to TFE3negative EHEs, which typically contained multiple lesions of smaller sizes. These data, together with the above findings, have newly established two clinically distinct subgroups of EHEs: TFE3-positive and TFE3-negative EHEs, which can be used to more accurately diagnose and treat EHEs in the future.

\section{MATERIALS AND METHODS}

\section{Cases}

This retrospective study was approved by the Institutional Review Board of Yonsei University Medical Center (approval number: 4-2014-0852). Our inclusion criteria defined a study population of 18 EHE patients who had been histologically diagnosed between 19932013. Archival tissues stained with hematoxylin and eosin (H\&E) were reviewed by three pathologists (SK Kim, SJ Lee, and WI Yang).

\section{Immunohistochemistry}

Tumors were fixed in formalin and embedded in paraffin. Briefly, $5 \mu \mathrm{m}$-thick sections were cut using a microtome, transferred onto adhesive slides, and dried at $62^{\circ} \mathrm{C}$ for $30 \mathrm{~min}$. Immunohistochemistry with antibodies against CD31 (M0823, Dako, CA, USA), CD34 (M7165, Dako, CA, USA), TFE3 (MRQ-37, CA, USA), and CAMTA1 (ab64119, Abcam, Cambridge, UK) was performed using an automated immunohistochemical staining instrument (Ventana Discovery ${ }^{\circledR}$ XT, Ventana Medical System, AZ, USA).

\section{Fluorescent in situ hybridization (FISH) assay}

FISH was performed on tumor sections after examination by H\&E microscopy. A ZytoLight SPEC TFE3 Dual Color Break-Apart Probe (Z-2109-200, ZytoVision $\mathrm{GmbH}$, Bremerhaven, Germany) and a MacProbe $^{\text {TM }}$ CAMTA1 Dual Color Break-Apart Probe (KH01M2NA12 and KH02M2NA12, Macrogen, South Korea) were used according to the manufacturer's instructions for the detection of translocations involving the TFE3 gene at Xp11.23 and the CAMTA1 gene at 1p36.23, respectively. Break-apart on the slides was evaluated using an epifluorescence microscope (Olympus, Tokyo, Japan). During interphase in normal cells without translocations, green/orange fusion signals appear. Xp11.23 or 1p36.23 loci affected by translocations are indicated by separate and distinct green and orange signals. A case was interpreted as positive when at least 
10 out of 50 counted tumor cells (20\%) showed separated green and orange signals [7].

\section{Reverse transcription polymerase chain reaction (RT-PCR)}

Isolation of RNA from formalin-fixed, paraffinembedded (FFPE) samples was performed following the manufacturer's protocol (RNeasy FFPE kit, Qiagen, Valencia, CA). RT-PCR was performed as previously published [3, 6, 7] using the following primers to identify the WWTR1-CAMTA1 fusion transcript or the YAP1-TFE3 fusion transcript: WWTR1 exon 4 forward, 5'-CCGTCAGTTCCACACCAGTGCCTC-3'; CAMTA1 exon 9 reverse, 5'-GGGGCTACAGCAGGGGAGGC-3'; YAP1 exon 1 forward, 5'-CCTGGAGGCGCTCTTGAACG-3'; TFE3 exon 6 reverse, 5'-GTTGCTGACAGTGATGGCTGG3'. The RT-PCR products were analyzed by electrophoresis.

\section{Statistics}

All statistical analyses were performed using GraphPad Prism 5 software, version 5.01 (GraphPad Software Inc., La Jolla, CA, USA) and SPSS for Windows, version 20.0 (SPSS Inc., Chicago, IL, USA). For the analysis of age at diagnosis and tumor size, a significant difference between means was determined by t-tests. Sex, tumor multiplicity, and metastasis were compared by Chisquare analyses and Fisher's exact tests.

\section{ACKNOWLEDGMENTS}

This work was supported by the Yonsei University New Faculty Research Seed Money Grant, 2015 (Grant number: 2015-32-0017).

\section{CONFLICTS OF INTERESTS}

The authors declare that they have no conflicts of interest in the article.

\section{REFERENCES}

1. Weiss SW, Antonescu CR, Bridge JA and Deyrup AT. (2013). WHO Classification of Tumours (Maestro 38330 Saint-Ismier, France: International Agency for Research on Cancer (IARC)).

2. Deyrup AT, Tighiouart M, Montag AG and Weiss SW. Epithelioid hemangioendothelioma of soft tissue: a proposal for risk stratification based on 49 cases. The American journal of surgical pathology. 2008; 32:924-927.

3. Errani C, Zhang L, Sung YS, Hajdu M, Singer S, Maki RG, Healey JH and Antonescu CR. A novel WWTR1-CAMTA1 gene fusion is a consistent abnormality in epithelioid hemangioendothelioma of different anatomic sites. Genes, chromosomes \& cancer. 2011; 50:644-653.

4. Mendlick MR, Nelson M, Pickering D, Johansson SL, Seemayer TA, Neff JR, Vergara G, Rosenthal H and Bridge JA. Translocation $\mathrm{t}(1 ; 3)(\mathrm{p} 36.3 ; \mathrm{q} 25)$ is a nonrandom aberration in epithelioid hemangioendothelioma. The American journal of surgical pathology. 2001; 25:684-687.

5. Tanas MR, Sboner A, Oliveira AM, Erickson-Johnson MR, Hespelt J, Hanwright PJ, Flanagan J, Luo Y, Fenwick K, Natrajan R, Mitsopoulos C, Zvelebil M, Hoch BL, Weiss SW, Debiec-Rychter M, Sciot R, et al. Identification of a disease-defining gene fusion in epithelioid hemangioendothelioma. Science translational medicine. 2011; 3:98ra82.

6. Antonescu CR, Le Loarer F, Mosquera JM, Sboner A, Zhang L, Chen CL, Chen HW, Pathan N, Krausz T, Dickson BC, Weinreb I, Rubin MA, Hameed M and Fletcher CD. Novel YAP1-TFE3 fusion defines a distinct subset of epithelioid hemangioendothelioma. Genes, chromosomes \& cancer. 2013; 52:775-784.

7. Flucke U, Vogels RJ, de Saint Aubain Somerhausen $\mathrm{N}$, Creytens DH, Riedl RG, van Gorp JM, Milne AN, Huysentruyt CJ, Verdijk MA, van Asseldonk MM, Suurmeijer AJ, Bras J, Palmedo G, et al. Epithelioid Hemangioendothelioma: clinicopathologic, immunohistochemical, and molecular genetic analysis of 39 cases. Diagnostic pathology. 2014; 9:131.

8. Shibuya R, Matsuyama A, Shiba E, Harada H, Yabuki K and Hisaoka M. CAMTA1 is a useful immunohistochemical marker for diagnosing epithelioid haemangioendothelioma. Histopathology. 2015;67:827-835.

9. Kanai F, Marignani PA, Sarbassova D, Yagi R, Hall RA, Donowitz M, Hisaminato A, Fugiwara T, Ito Y, Cantley LC, and Yaffe MB. TAZ: A novel transcriptional coactivator regulated by interactions with 14-3-3 and PDZ domain proteins. The EMBO Journal. 2000;19:6778-6791.

10. Tanas MR, Ma S, Jadaan FO, Ng CK, Weigeit B, ReisFilho JS, and Rubin BP. Mechanism of action of a WWTR1(TAZ)-CAMTA1 fusion oncoprotein. Oncogene. 2015 May 11. doi: 10.1038/onc.2015.148. PMID: 25961935.

11. Patel NR, Salim AA, Sayeed H, Sarabia SF, Hollingsworth F, Warren M, Jakacky J, Tanas M, Oliveira AM, Rubin BP, Lazar AJ, Lopez-Terrada D, and Wang WL. Molecular characterization of epithelioid haemangioendotheliomas identifies novel WWTR1-CAMTA1 fusion variants. Histopathology. 2015;67:699-708.

12. Taylor BS, Barretina J, Maki RG, Antonescu CR, Singer S, and Ladanyi M. Advances in sarcoma genomics and new therapeutic targets. Nature Reveiws Cancer. 2011;11:541557.

13. Borden EC, Baker LH, Bell RS, Bramwell V, Demetri GD, Eisenberg BL, Fletcher CD, Fletcher JA, Ladanyi M, Meltzer P, O'Sullivan B, Parkinson DR, Pisters PW, et al. 
Soft tissue sarcomas of adults: state of the translational science.Clinical Cancer Research. 2003;9:1941-1956

14. Helman, L. J. and Meltzer, P. Mechanisms of sarcoma development. Nature Reviews Cancer. 2003;3:685-694. 